\title{
Research Progress on the Mechanism and Preventive Measures of Diabetes Induced Peri-implantitis
}

\author{
Wanyun Lin ${ }^{1}$, Chen $\mathrm{Xie}^{1}$, Chunyi $\mathrm{Cao}^{2}$, Shengping $\mathrm{Cao}^{3}$, Xiaoting $\mathrm{Li}^{4}$, Tingting $\mathrm{Li}^{3}$, \\ Yujia Zhai ${ }^{3}$, Jingni Guo ${ }^{1}$, Vannesa Bongani Muyeleka ${ }^{3}$, Zhuling Guo ${ }^{1,2, *}$ \\ ${ }^{1}$ School of Dentistry, Hainan Medical University, Haikou, PR China \\ ${ }^{2}$ Hainan Eye Hospital and Key Laboratory of Ophthalmology, Zhongshan Ophthalmic Center, Sun Yat-sen University, Guangzhou, PR China \\ ${ }^{3}$ School of Clinical Medicine, Hainan Medical University, Haikou, PR China \\ ${ }^{4}$ Department of Dentistry, the First Affiliated Hospital of Hainan Medical University, Haikou, PR China
}

Email address:

604569033@qq.com (Zhuling Guo)

${ }^{*}$ Corresponding author

\section{To cite this article:}

Wanyun Lin, Chen Xie, Chunyi Cao, Shengping Cao, Xiaoting Li, Tingting Li, Yujia Zhai, Jingni Guo, Vannesa Bongani Muyeleka, Zhuling Guo. Research Progress on the Mechanism and Preventive Measures of Diabetes Induced Peri-implantitis. International Journal of Dental Medicine. Vol. 7, No. 1, 2021, pp. 10-14. doi: 10.11648/j.ijdm.20210701.12

Received: April 28, 2021; Accepted: May 17, 2021; Published: May 27, 2021

\begin{abstract}
Background: Diabetes mellitus has been shown to be an associated risk factor for peri-implantitis. Peri-implantitis has become the leading cause of implant failure. Objective: To ensure the postoperative efficacy of diabetic patients and avoid the occurrence of peri-implantitis, research progress on the mechanism and preventive measures of diabetes induced peri-implantitis were elaborated. Methods: With "diabetes mellitus or diabetes or DM, peri-implantitis, implant, osseointegration" as the search terms, related articles published during 2013-2021 in PubMed, CNKI, ScienceDirect and other databases were searched. Results: The changes in the type and proportion of bacteria around implants in diabetic patients break the balance between bacteria and host defense system. Diabetic patients with insulin resistance and persistent hyperglycemia inhibit osteoblast differentiation, induce osteoclast activation and make adverse effects on osseointegration. The immune inflammatory state and local microvascular lesions of the body slow down postoperative tissue healing and induce peri-implantitis. According to the relevant mechanisms, many experiments have achieved good results in reducing the incidence of peri-implantitis in patients with diabetes through plaque control, blood glucose management and implant interface improvement. Conclusion: Diabetic patients have a higher risk of peri-implantitis, so implant patients with diabetes mellitus should undertake related prevention and treatment to reduce the risk of peri-implantitis.
\end{abstract}

Keywords: Diabetes Mellitus, Implant, Peri-Implantitis, Osseointegration

\section{Introduction}

Dental implant repair is the best treatment for missing teeth, but local and systemic risk factors can lead to implant failure. Diabetes mellitus (DM) is a chronic disease with hyperglycemia and many side effects. It can be divided into non-insulin-dependent, type-1diabetes Mellitus (T1DM) and insulin-dependent, type-2 diabetes mellitus (T2DM) clinically. The number of patients with diabetes in the world is expected to rise to 629 million in 2045 [1], of which T2DM is more common. Periodontal disease is the sixth major complication of diabetes mellitus. The tooth loss rate of patients with diabetes mellitus and periodontitis is more than twice the average level. Dental implant repair has gradually become the best choice for diabetic patients with periodontal disease after tooth loss. Diabetes mellitus as a relative contraindication of implant surgery has been controversial.

Diabetes has a negative impact on the implant, which can inhibit the healing rate of oral mucosa and alveolar bone wound, and is prone to cause microvascular thrombosis, which makes it easy to infect. On the other hand, diabetes will also affect the osseointegration of dental implants, which makes dental implants easy to loose and fall off, and the failure rate is higher than that of non-diabetic patients. 
Diabetes mellitus is a risk factor for peri-implantitis and a common postoperative complication in patients with diabetes mellitus after implant surgery. Peri-implantitis is one of the main causes of implant failure. Peri-implantitis is an inflammatory disease accumulated around the implant, which has the characteristics of soft tissue inflammatory lesions and loss of marginal supporting bone. The clinical manifestations of peri-implantitis include hyperemia, redness, swelling, bleeding on probing, and pus overflow is more common, accompanied by progressive bone resorption.

\section{Diagnostic Criteria of Peri-Implantitis}

There were obvious plaque or calculus around the implant and abutment, mucosa redness and swelling, probing bleeding and (or) pus overflow and depth $\geq 6 \mathrm{~mm}$. X-ray showed the horizontal or vertical bone absorption around the implant $\geq 3$ $\mathrm{mm}$, or transmission shadow between the implant and bone tissue [2-4].

\section{Prevalence of Peri-Implantitis in Diabetic Patients}

Two cross-sectional studies showed that the relative risk of peri-implantitis due to diabetes was 1.9 and 4.1, respectively [5] Some local surveys, such as North Africa, showed that the prevalence of peri-implant mucositis and peri-implant inflammations were $82.1 \%$ and $42.4 \%$ respectively, $68.4 \%$ and $22.7 \%$ respectively. Diabetes was positively correlated with these two results [6]. Another statistical study showed that the incidence of post-implant complications was low in patients without diabetes mellitus. With the increase of glycosylated hemoglobin (HbA1c) level, the probing depth and blood loss around the implant increased in patients with diabetes mellitus, that is, the possibility of peri-implantitis increased [7]. According to a 7-year clinical observation, the study showed that the clinical manifestations of peri-implantitis in patients with T2DM were significantly higher than those in non-diabetic patients [8]; the risk of peri-implantitis in diabetic patients is $50 \%$ higher than that in non-diabetic patients, and the risk of osseointegration damage and peri-implantitis in diabetic patients with poor glycemic control is increased [9].

\section{The Mechanism of Peri-Implantitis in Diabetic Patients}

\subsection{Microorganism}

Peri-implantitis is the result of the interaction between bacteria and the immune function of periodontal tissue. The flora around the successful implant is very similar to the adjacent natural teeth. The bacteria can appear soon after the implant implantation, mainly gram-positive cocci, and there is no significant change in the flora six months after the implant implantation [10]. A large number of studies have proved that the subgingival is a complex and unique micro ecological environment when peri-implantitis occurs. Gram-negative anaerobes are the main bacteria around the implant. In addition to the "Red complex" (Porphyromonas gingivalis, Treponema denticola, and forsythia) and "orange complex" (fusobacteria and Prevotella intermedia), Campylobacter virens and soft tissue bacteria can also be detected Phylum parietal, Enterococcus faecalis, etc [11-13]. At the same time, some studies have shown that the proportion of subgingival plaque species including cocci, bacilli, and fusiform bacteria in T2DM patients after implantation is higher than that in non-diabetic patients [14]. This indicates that the subgingival flora around implants in diabetic patients significantly increases the probability of peri-implantitis.

\subsection{Diabetes and Osseointegration}

Branemark proposed the concept of osseointegration, that is, the implant embedded in the living bone directly contacts with the bone tissue under the light microscope. And there is no tissue other than bone tissue, such as connective tissue. This is the biological basis of oral implantology. Based on the theory of osseointegration, implantology has developed rapidly. Nowadays, dental implant prosthesis is more and more accepted by Stomatologists and patients because of its wide range of indications, beautiful and comfortable, good retention, and support. The basic steps of the oral implant include (take two-stage two-step bone implant as an example): for patients with indications for implant restoration, implant retention nail is implanted into the alveolar bone in the edentulous area through a one-stage operation. After 3-4 months of implant osseointegration, it is feasible to install the healing abutment connecting with gingival tissue and finally complete the implant restoration. In this process, the formation of good osseointegration is a necessary basic condition.

In the study of rats, diabetes can affect the process of osseointegration after implantation [15]. Diabetic patients will affect the stability of implants by influencing the repair of periodontal bone tissue [16]; Some clinical studies have shown that the bone loss around implants in diabetic patients is more obvious than that in non-diabetic patients after implantation, which may be related to the state of immune inflammation [17]. At the same time, some studies have shown that poor blood glucose control can negatively regulate bone factors in the process of implant healing [18]. At present, the influence mechanism of DM on osseointegration of implants is as follows, which needs to be further improved.

\subsubsection{Hyperglycemia}

Hyperglycemia can inhibit osteoblast differentiation and promote osteoclast formation. Hyperglycemia can directly affect bone binding by inhibiting the osteogenesis of bone marrow mesenchymal stem cells (BMSC) mediated by reactive oxygen species [19]. Hyperglycemia can inhibit the formation of mineralized nodules of BMSC, and induce osteogenic differentiation genes such as alkaline phosphatase (ALP), osteocalcin (OC), and collagen type I through MAPK, PI3K/Akt, Wnt/ $/$-catenin, and Notch pathways 1 . The expression of CoL-I decreased, which reduced the function 
and number of osteoblasts and then affected osseointegration [20]. In addition, long-term hyperglycemia can lead to the production of advanced glycation end products (AGEs) through nonenzymatic glycosylation of a large number of proteins in the body. AGE in the gingival crevicular fluid around implants increases with the increase of blood glucose in T2DM patients [21]. A High concentration of AGE combined with its receptor of AGEs (RAGE) can inhibit osteoblast differentiation and induce osteoclast activation through the mitogen- activated protein kinase (MAPK) pathway, resulting in poor bone binding.

\subsubsection{Diabetes and Immune Inflammatory Process}

Diabetic patients are in the abnormal metabolic state of long-term hyperglycemia, which will increase the chance of infection around the implant [22]. Oxidative stress plays an important role in the process of diabetes. For example, reactive oxygen species (ROS) increase in the body. Under the induction of ROS, nuclear factor $-\kappa \mathrm{B}(\mathrm{NF}-\kappa \mathrm{B})$ was activated, which promoted the secretion of interleukin-1 $\beta$ (IL-1 $\beta)$ and interleukin-8(IL-8) [23, 24]. IL-8 can induce neutrophils to chemotaxis and move out of the epithelium in the tissue around the implant. During the process of phagocytizing bacteria, IL-8 can produce superoxide ions and release lysosomal enzyme cytokines, which will aggravate the inflammation of tissue around the implant, reduce the collagen and extracellular matrix in periodontium and the tissue around the implant, and slow down the postoperative tissue healing. Once neutrophil chemotaxis and phagocytosis are destroyed, it will weaken the host's barrier function and immune defense function against periodontal pathogens, and promote the occurrence of peri-implantitis. In addition, a large number of experiments have confirmed that IL-17 is overexpressed in peri-implantitis gingival crevicular fluid and T2DM patients [25]. It is suggested that IL-17 may be the signal pathway of peri-implantitis and T2DM. At the same time, the increase of IL-6 has been confirmed as an independent predictor of T2DM, and the increase of IL- 6 in peri implant crevicular fluid is considered as a marker of peri-implantitis.

\subsubsection{Insulin Resistance (IR)}

Insulin can not only regulate the concentration of blood glucose, but also bind to the insulin receptor on the surface of osteoblasts. It directly stimulated the differentiation and proliferation of osteoblasts, and promote the osseointegration after transplantation. However, T1DM patients are caused by destruction of islet B cells, resulting in insufficient or absolute lack of insulin secretion; T2DM patients are caused by insulin resistance or progressive insufficient insulin secretion. If the body has insulin resistance, it can promote the increase of tumor necrosis factor- $\alpha(\mathrm{TNF}-\alpha)$ through the endocrine and paracrine of cytokines. Some in vitro and animal experiments have proved that TNF- $\alpha$ can inhibit the proliferation and differentiation of osteoblasts, promote their apoptosis. And it can activate osteoclasts to inhibit new bone formation [26]. Moreover, insulin deficiency in DM patients leads to the decrease of 1,25-(OH)2-D3 synthesis. The decrease of osteopontin secreted by $1,25-(\mathrm{OH}) 2-\mathrm{D} 3$, which leads to the increase of osteoclast activity and the decrease of osseointegration rate and success rate [27]. The decrease of osteoblast differentiation and proliferation and the increase of osteoclast activity are not conducive to osseointegration.

\subsection{Microangiopathy}

Vascular disease is a common complication of DM patients. Vascular endothelial cell dysfunction and abnormal coagulation mechanism lead to capillary basement membrane thickening, lumen stenosis, slow blood flow, and reduced nutrition supply to local cells and tissues. This is not conducive to the metabolism of bone tissue around implants [28].

\section{Prevention and Treatment of Peri-Implantitis in Diabetic Patients}

\subsection{Plaque Control}

Because plaque aggregation is the key factor of peri-implantitis, controlling bacteria is the core of the prevention and treatment of peri-implantitis. Surgical treatment includes mechanical debridement, implant surface decontamination, bone transplantation, and biological media. Non-operative treatment includes ultrasonic curettage, adjuvant drug therapy, antibacterial photodynamic therapy, and combination therapy. For example, washing with chlorphenamine and the local application of minocycline hydrochloride can significantly improve the bone defect around the implant [29]. Antibacterial photodynamic therapy and decontamination procedures are expected to be developed in the future [30].

\subsection{Blood Glucose Management}

Increased bone loss around implants is associated with higher glycosylated glycemic protein levels [31]. It is very necessary to control blood glucose before and after implant surgery, which can improve the osseointegration rate and promote recovery after implant surgery. Insulin can be used as a direct and effective way to control blood glucose, inhibit osteoclasts, promote osteoblasts, and increase implant bone-bonding; metformin, a commonly used drug for the treatment of T2DM, can also reverse the negative effect of hyperglycemia on implant-bone integration [32]; some animal studies have shown that insulin combined with VD (3) treatment may be an effective way to improve implant fixation in diabetic rats [33].

\subsection{Implant Interface Improvement}

In recent years, many experiments focus on the improvement of implant interface in diabetic patients, which may be a way to promote implant osseointegration in diabetic patients in the future. It has been proved in animal experiments that chitosan-coated porous titanium alloy can promote bone integration by inhibiting local redox reaction under diabetic conditions [34]. What's more, in vitro studies have shown that in peri-implantitis, the exposed implant surface is coated with platelet-rich fibrin and etched with 
phosphoric acid, so as to improve the blood wettability of the implant surface and facilitate the healing of tissue [35].

\section{Conclusion}

Diabetic patients have a higher risk of peri-implantitis, so implant patients with diabetes mellitus should undertake related prevention and treatment to reduce the risk of peri-implantitis. However, the mechanism of diabetes induced peri-implantitis is not yet clear, and the prevention and treatment methods need to be further improved.

\section{Fund}

This research was funded by Education Department of Hainan Province (project number: Hnjg2021-60), Course Construction Project of Hainan Medical University (HYJW202117), Educational Scientific Research of Hainan Medical University (HYYB202014), Marxism Theory Discipline Research Project of Hainan Medical University (QMYKYLX201807), Research Project of Hainan Provincial Humanities Medical Research Base (QRYZH201811 (YB)), Innovative Entrepreneurial Training Program of Hainan Medical University (S201911810026).

\section{References}

[1] Ogurtsova K, da Rocha Fernandes JD, Huang Y, et al. IDF Diabetes Atlas: global estimates for the prevalence of diabetes for 2015 and 2040 [J]. Diabetes Res Clin Pract, 2017, 128: 40-50.

[2] Berglundh T, Armitage G, Araujo MG, et al. Peri-implant diseases and conditions: Consensus report of workgroup 4 of the 2017 World Workshop on the Classification of Periodontal and Peri-Implant Disease and condition [J]. Clin Periodontol, 2018, 45 (S20): S286-S291.

[3] Mario Romandini, Cristina Lima, Ignacio Pedrinaci, et al. Prevalence and risk/protective indicators of peri-implant diseases: A university-representative cross-sectional study [J]. 2020 .

[4] Monje Alberto, Vera Maria, MuñozSanz Agustín, et al. Suppuration as diagnostic criterium of peri-implantitis [J]. Journal of Periodontology, 2020, 92 (2): 216-224.

[5] Daubert DM, Weinstein BF, Bordin S, et al. Prevalence and predictive factors for peri-implant disease and implant failure: a cross-sectional analysis [J]. Periodontol, 2015, 86 (3): 337347.

[6] Kissa Jamila, Kholti Wafa El, Chemlali Sihame, et al. Prevalence and risk indicators of peri-implant diseases in a group of Moroccan patients [J]. Journal of Periodontology, 2020. Doi: 10.1002/JPER.20-0549.

[7] Jiang Xue, Zhu Yanlin, Liu Zhaoying, et al. Association between diabetes and dental implant complications: a systematic review and meta-analysis [J]. Acta Odontologica Scandinavica, 2021, 79 (1): 9-18.
[8] Alqahtani F, Alqhtani N, Alkhtani F, et al. Clinicoradiographic markers of peri-implantitis in cigarette-smokers and never-smokers with type 2 diabetes mellitus at 7-years follow-up [J]. Journal of Periodontology, 2020, 91 (6).

[9] Naujokat H, Kunzendorf B, Wiltfang J. Dental implants and diabetes mellitus-a systematic review [J]. International Journal of Implant Dentistry, 2016, 2 (5). Doi: https://doi.org/10.1186/s40729-016-0038-2.

[10] Moran G. An in vivo comparison of bacterial microleakage in two dental implant systems: identification of a pathogenic reservoir in dental implants [J]. Acta Odontologica Scandinavica, 2015, 73 (3): 188-194.

[11] Tenenbaum $H$, Bogen $O$, Séverac $F$, et al. Long-term prospective cohort study on dental implants: clinical and microbiological parameters [J]. ClinO ral Implants Res, 2017, 28 (1): 86-94.

[12] Flanagan D. Enterococcus faecalis and Dental Implants [J]. J Oral Implantol, 2017, 43 (1): 8-11.

[13] Robitaille N, Reed DN, Walters JD, et al. Periodontal and Peri-implant Diseases: Identical or Fraternal Infections? [J]. Mol Oral Microbiol, 2016, 31 (4): 285-301.

[14] Miranda Tamires, Feres Magda, Retamal-Valdés Belén, et al. Influence of glycemic control on the levels of subgingival periodontal pathogens in patients with generalized chronic periodontitis and type 2 diabetes [J]. Journal of Applied Oral Science, 2017, 25 (1): 82-89.

[15] Oliveira Paula, Coelho Paulo, Bergamo Edmara, et al. Histological and Nanomechanical Properties of a New Nanometric Hydroxiapatite Implant Surface. An In Vivo Study in Diabetic Rats [J]. Materials, 2020, 13 (24): 5693.

[16] Monje A, Catena A, Borgnakke W. Association between Diabetes Mellitus/Hyperglycemia and Peri-Implant Diseases: Systematic Review and Meta-Analysis [J]. Journary Clinical peridontonology, 2017, 44 (6): 636-648.

[17] Saeed AZ, Abdulaziz A. Stability and bone loss around submerged and non-submerged implants in diabetic and non-diabetic patients: a 7-year follow-up [J]. Brazilian oral research, 2018, 32: e57.

[18] Ghiraldini, Bruna, Conte, et al. Influence of Glycemic Control on Peri-Implant Bone Healing: 12-Month Outcomes of Local Release of Bone-Related Factors and Implant Stabilization in Type 2 Diabetics [J]. Clinical Implant Dentistry \& Related Research, 2016, 18 (4): 801-809.

[19] GAO Hai, CHEN Xiao, GUAN Donghua, et al. Effects of high glucose on osteogenic differentiation of hBMSC [J]. Prevention and control of oral diseases, 2017, 25 (1): 26-30.

[20] Yu Tianliang, Acharya Aneesha, Mattheos Nikos, et al. Molecular mechanisms linking peri-implantitis and type 2 diabetes mellitus revealed by transcriptomic analysis [J]. PeerJ, 2019, (7): e7124.

[21] Al-Sowygh ZH, Ghani SMA, Sergis K, et al. Peri-implant conditions and levels of advanced glycation end products among patients with different glycemic control [J]. Clin Implant Dent Relat Res, 2018, 20 (3): 345-351.

[22] Passi D, Singh M, Dutta S R, et al. Newer proposed classification of periimplant defects: A critical update $[\mathrm{J}]$. J O-ral Biol Craniofac Res, 2017, 7 (1): 58-61. 
[23] Nguyen Quynh, Fang Minzhe, Zhang Mengyang, et al. Crataegus laevigata Suppresses LPS-Induced Oxidative Stress during Inflammatory Response in Human Keratinocytes by Regulating the MAPKs/AP-1, NFKB, and NFAT Signaling Pathways [J]. Molecules, 2021, 26 (4): 869-869.

[24] H-C, Chiu, et al. Effect of high glucose, Porphyromonas gingivalis lipopolysaccharide and advanced glycation end-products on production of interleukin- $6 /-8$ by gingival fibroblasts [J]. Journal of Periodontal Research, 2017, 52 (2): 268-276.

[25] Nadeem A, Javaid K, Sami W, et al. Inverse relationship of serum IL-17 with type-II diabetes retinopathy [J]. Clinical Laboratory, 2013, 59: 1311-1317.

[26] Ko Kang, Coimbra Leila, Tian Chen, et al. Diabetes reduces mesenchymal stem cells in fracture healing through a TNF $\alpha$-mediated mechanism [J]. Diabetologia, 2015, 58 (3): 633-642.

[27] Algate K, Haynes DR, Bartold PM, et al. The effects of tumour necrosis factor- $\alpha$ on bone cells involved in periodontal alveolar bone loss; osteoclasts, osteoblasts and osteocytes [J]. J Periodont Res, 2016, 51 (5): 549-566.

[28] Shanbhogue VV, Hansen S, Frost M, et al. Bone disease in diabetes: another manifestation of microvascular disease? [J]. Lancet Diabetes\&Endocrinology, 2017, 5 (10): 827-838.

[29] Heo SunJin, Kim Hyun-Joo, Joo Ji-Young, et al. Simplified nonsurgical treatment of peri-implantitis using chlorhexidine and minocycline hydrochloride [J]. Journal of Periodontal \& Implant Science, 2018, 48 (5): 326-333.

[30] Seigo Ohba, Mika Sato, Sawako Noda, et al. Assessment of safety and efficacy of antimicrobial photodynamic therapy for peri-implant disease [J]. Photodiagnosis and Photodynamic Therapy, 2020, 31.

[31] Aguilar-Salvatierra Antonio, Calvo-Guirado José Luis, González-Jaranay Maximino, et al. Peri-implant evaluation of immediately loaded implants placed in esthetic zone in patients with diabetes mellitus type 2: a two-year study [J]. Clinical Oral Implants Research, 2016, 27 (2): 156-161.

[32] Serrão Caroline Ribeiro, Bastos Marta Ferreira, Cruz Daniele Ferreira, et al. Role of Metformin in Reversing the Negative Impact of Hyperglycemia on Bone Healing Around Implants Inserted in Type 2 Diabetic Rats [J]. International Journal of Oral \& Maxillofacial Implants, 2017, 32 (3): 547-554.

[33] Wu Yingying, Yu Tao, Yang Xiaoyong, et al. Vitamin D3 and insulin combined treatment promotes titanium implant osseointegration in diabetes mellitus rats [J]. Bone, 2013, 52 (1): $1-8$

[34] Wang L, Li X. Osseointegration Of Chitosan Coated Porous Titanium Alloy Implant By Reactive Oxygen Species-mediated Activation of The Pi3k/akt Pathway Under Diabetic Conditions [J]. Tissue Engineering Part A, 2017, 23: S92-S92.

[35] Bevilacqua Lorenzo, Faccioni Francesco, Porrelli Davide, et al. Blood Wettability of Different Dental Implant Surfaces after Different Pre-Treatments: Ultrasonic Instrumentation, Platelet-Rich Fibrin Coating, and Acid Etching. An In Vitro Study [J]. Applied Sciences, 2021, 11 (4): 1433-1433.

[36] Saeed Al Zahrani, Abdullah A Al Mutairi. Crestal Bone Loss Around Submerged and Non-Submerged Dental Implants in Individuals with Type-2 Diabetes Mellitus: A 7-Year Prospective Clinical Study [J]. Medical Principles and Practice, 2019, 28 (1): 75-81.

[37] Cirano FR, Pimentel SP, Casati MZ, et al. Effect of curcumin on bone tissue in the diabetic rat: repair of peri-implant and critical-sized defects $[\mathrm{J}]$. International Journal of Oral and Maxillofacial Surgery, 2018, 47 (11): 1495-1503.

[38] Paulo, Coelho, Benjamin, et al. Obesity/Metabolic Syndrome and Diabetes Mellitus on Peri-implantitis [J]. Trends in Endocrinology \& Metabolism, 2020, 31 (8): 596-610. 